\title{
Brownian dynamics investigation of the Boltzmann superposition principle for orthogonal superposition rheology
}

Cite as: J. Chem. Phys. 150, 014903 (2019); https://doi.org/10.1063/1.5080333

Submitted: 07 November 2018 . Accepted: 17 December 2018. Published Online: 04 January 2019

Vishal Metri (D), and W. J. Briels (D)
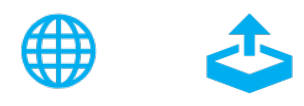

View Online

Export Citation

\section{ARTICLES YOU MAY BE INTERESTED IN}

Leading JCP into the future

The Journal of Chemical Physics 150, 010401 (2019); https://doi.org/10.1063/1.5085674

Understanding non-covalent interactions in larger molecular complexes from first principles

The Journal of Chemical Physics 150, 010901 (2019); https://doi.org/10.1063/1.5075487

Revisiting the Stokes-Einstein relation without a hydrodynamic diameter

The Journal of Chemical Physics 150, 021101 (2019); https://doi.org/10.1063/1.5080662

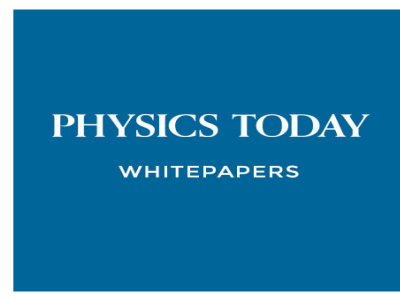

ADVANCED LIGHT CURE ADHESIVES

Take a closer look at what these

environmentally friendly adhesive systems can do
READ NOW

PRESENTED BY 8 MASTERBOND" 


\title{
Brownian dynamics investigation of the Boltzmann superposition principle for orthogonal superposition rheology
}

\author{
Cite as: J. Chem. Phys. 150, 014903 (2019); doi: 10.1063/1.5080333 \\ Submitted: 7 November 2018 - Accepted: 17 December 2018 • \\ Published Online: 4 January 2019
}

\begin{abstract}
Vishal Metri ${ }^{1,2, a)}$ (D) and W. J. Briels $\mathbf{s}^{1,2,3, b)}$ (D)

\section{AFFILIATIONS}

${ }^{1}$ Computational Chemical Physics, Faculty of Science and Technology, University of Twente, P.O. Box 217, 7500 AE Enschede, The Netherlands

${ }^{2}$ MESA+ Institute for Nanotechnology, University of Twente, P.O. Box 217, 7500 AE Enschede, The Netherlands

${ }^{3}$ Forschungszentrum Jülich, ICS 3, Wilhelm-Johnen-Straße, 52428 Jülich, Germany

a)Electronic mail: v.metri@utwente.nl

b) Electronic mail: w.j.briels@utwente.nl

ABSTRACT

The most general linear equation describing the stress response at time $t$ to a time-dependent shearing perturbation may be written as the integral over the past history $t^{\prime}$ of a time dependent relaxation modulus, depending on $t-t^{\prime}$, multiplied by the perturbing shear rate at time $t^{\prime}$. This is in agreement with the Boltzmann superposition principle, which says that the stress response of a system to a time dependent shearing deformation may be written as the sum of responses to a sequence of stepstrain perturbations in the past. In equilibrium rheology, the Boltzmann superposition principle gives rise to the equality of the shear relaxation modulus, obtained from oscillatory experiments, and the stress relaxation modulus measured after a step-strain perturbation. In this paper, we describe the results of Brownian dynamics simulations of a simple soft matter system showing that the same conclusion does not hold when the system is steadily sheared in a direction perpendicular to the probing flows, and with a gradient parallel to that of the probing deformations, as in orthogonal superposition rheology. In fact, we find that the oscillatory relaxation modulus differs from the step-strain modulus even for the smallest orthogonal shear flows that we could simulate. We do find, however, that the initial or plateau levels of both methods agree and provide an equation relating the plateau value to the perturbation of the pair-function.
\end{abstract}

Published under license by AIP Publishing. https://doi.org/10.1063/1.5080333

\section{INTRODUCTION}

The non-linear flow properties of complex fluids like polymers, colloidal suspensions, surfactants, etc., are not fully understood and are the subject of much discussion. One of the techniques of mechanical spectroscopy used to study such non-equilibrium phenomena, superposition rheology, has emerged in recent years as a very useful tool to probe soft matter systems far from equilibrium. ${ }^{1-10}$ In superposition techniques, two different shear flows are superimposed simultaneously on the sample under study. One of them is a steady shear that disturbs and defines the sample far from equilibrium, and the second is a small oscillation superimposed on the first, probing the sample. The first will be called "the disturbing flow" and the second "the probing flow." The probing flow is either in the direction of the disturbing flow, in which case the method is called Parallel Superposition Rheology (PSR), ${ }^{1,1-16}$ or orthogonal to it, when it is called Orthogonal Superposition Rheology (OSR).,17-19 PSR experiments are easier to perform than OSR experiments; hence, there have been a substantial number of studies published that are dedicated to PSR. ${ }^{12-15,20-25}$ Constitutive equations used to define system properties both under PSR and OSR conditions, however, have been found to be much more difficult to apply to 
PSR data than to OSR data. 2,26,27 This is because the small amplitude shear used to probe the material has the same symmetry as the disturbing flow that defines the material. Hence, in this study, we will concentrate on OSR only, aiming for some understanding of the out-of-equilibrium material properties in a way similar to that achieved with linear visco-elastic properties.

With the construction of OSR apparatus by Vermant and others providing reliable and reproducible data, $2,28,29 \mathrm{sev}-$ eral experiments have been done on a variety of soft matter systems. Most of these experiments have been analyzed using Yamamoto's phenomenological expressions. ${ }^{17,27,30-32}$ Yamamoto defines a generalized response spectrum $\mathrm{H}(\tau, \dot{\gamma})$ which depends on the shear rate $\dot{\gamma}$ but is otherwise related to the storage and loss moduli in the same way as in linear viscoelasticity near equilibrium. Among other things this implies the validity of the Kramers-Kronig relations ${ }^{33}$ as already noticed by Vermant et al. ${ }^{2}$ and Dhont and Wagner. ${ }^{3}$ Other models have been suggested, like the K-BKZ based constitutive model by Tanner and Williams which expresses the storage and loss moduli in terms of some time dependent elastic energy and the Wagner model which gives slightly different relations for the relaxation moduli from those of Yamamoto. In addition, there is a mode coupling theory based analysis, ${ }^{34-36}$ relating the structure and rheology. Hence, there is a wide variety of constitutive models available in the literature that provide different kinds of interpretations of OSR data.

As emphasized above and explained in Sec. II below, the equations proposed by Yamamoto show a large resemblance to those used to analyze linear viscoelastic experiments near equilibrium. The main difference is that the response spectrum $\mathrm{H}(\tau, \dot{\gamma})$ depends on the imposed stationary shear rate $\dot{\gamma}$. A natural question then is how much of the conceptual framework of linear viscoelasticity near equilibrium may be transferred to OSR applied to systems brought far out of equilibrium by the stationary applied shear flow. One may ask if the usual Green-Kubo relations are still valid, or if the relationship between various different experimental techniques, like, for example, between step strain and oscillatory experiments, are still valid. The emphasis here must in principle be on "far out of equilibrium" because with small values of $\dot{\gamma}$ of the disturbing flow, the experiments degenerates into the superposition of two independent, mutually orthogonal linear experiments near equilibrium, in which case linear viscoelastic analysis applies. It is the main goal of this work to shed some light on the answers to these questions. The obvious method of choice in this case will be particle based simulations since they provide the most detailed information and allow for the most detailed analysis. Since OSR is of most interest to soft matter physicists, we choose to model a system of star polymers, characteristic both for polymer behavior and colloidal behavior. ${ }^{37}$ We use a single particle model in which the particles interact through a potential developed by Likos and co-workers ${ }^{38}$ and are propagated through Brownian dynamics.
To the best of our knowledge, no study similar to the one reported here has been published till now. Somewhat related work has been done by Jacob et al., ${ }^{18}$ but there the emphasis was on supporting experimental findings instead of interrogating fundamental assumptions about OSR. Besides this, stresses were not calculated directly, but were inferred from mean square displacements invoking the generalized Stokes-Einstein relation, ${ }^{39}$ which in itself is not fully undisputed.

This paper is structured as follows. In Sec. II, we present a bit of the theoretical background of the Yamamoto model and explain the context of the questions that we want to address. In Sec. III, we describe the system and the methods that we used for the simulation. In Sec. IV, we present the results of our simulations. In Sec. V, we summarize our findings. In Appendixes A and B, we present some derivations of theoretical expressions that need to be analyzed and discuss our results.

\section{THEORY}

We apply a perturbing shear flow in the $\mathrm{x}$-direction with the gradient in the $\mathrm{y}$-direction and an oscillatory probing shear flow in the $\mathrm{z}$-direction with the gradient in the $\mathrm{y}$ direction. Measurements are performed once all transients are over and the flow velocities are given by

$$
\begin{aligned}
& u_{x}(t)=\dot{\gamma} y, \\
& u_{y}(t)=0, \\
& u_{z}(t)=\gamma_{\perp} \omega \cos (\omega t) y,
\end{aligned}
$$

where $\dot{\gamma}$ is the imposed perturbing shear rate and $\gamma_{\perp}$ the amplitude of the probing flow. According to Yamamoto, the stress component $\sigma_{y z}(t ; \dot{\gamma})$ in this case is given by

$$
\sigma_{y z}(t ; \dot{\gamma})=\gamma_{\perp}\left[G_{\perp}^{\prime}(\omega ; \dot{\gamma}) \sin (\omega t)+G_{\perp}^{\prime \prime}(\omega ; \dot{\gamma}) \cos (\omega t)\right],
$$

with

$$
\begin{aligned}
& \mathrm{G}_{\perp}^{\prime}(\omega ; \dot{\gamma})=\int_{-\infty}^{\infty} \mathrm{H}(\tau, \dot{\gamma}) \frac{(\omega \tau)^{2}}{1+(\omega \tau)^{2}} \mathrm{~d} \ln \tau \\
& \mathrm{G}_{\perp}^{\prime \prime}(\omega ; \dot{\gamma})=\int_{-\infty}^{\infty} \mathrm{H}(\tau, \dot{\gamma}) \frac{\omega \tau}{1+(\omega \tau)^{2}} \mathrm{~d} \ln \tau .
\end{aligned}
$$

$\mathrm{H}(\tau, \dot{\gamma})$ is the aforementioned response spectrum, which in this case may depend on $\dot{\gamma}$. It is not difficult to show that these equations are equivalent to

$$
\sigma_{y z}(t ; \dot{\gamma})=\int_{-\infty}^{t} G_{\perp}\left(t-t^{\prime}, \dot{\gamma}\right) \dot{\gamma}_{\perp}\left(t^{\prime}\right) d t^{\prime}
$$

with $\dot{\gamma}_{\perp}(t)=\gamma_{\perp} \omega \cos (\omega t)$ and

$$
\mathrm{G}_{\perp}\left(\mathrm{t}-\mathrm{t}^{\prime}, \dot{\gamma}\right)=\int_{-\infty}^{\infty} \mathrm{H}(\tau, \dot{\gamma}) e^{-\left(\mathrm{t}-\mathrm{t}^{\prime}\right) / \tau} \mathrm{d} \ln \tau,
$$

and that under weak conditions on the response spectrum, the Kramers-Kronig relations must hold.

Of course Eq. (4) is the usual expression used in linear rheology near equilibrium to define the shear relaxation 
modulus. Near equilibrium, we put $\dot{\gamma}=0$, and we omit the superfluous subscript $\perp$. Equation (4) becomes $\sigma_{y z}(t)$ $=\int_{-\infty}^{t} \mathrm{G}\left(t-t^{\prime}\right) \dot{\gamma}_{y z}\left(t^{\prime}\right) \mathrm{d} t^{\prime}$, which is the most general linear expression possible in agreement with causality. Near equilibrium, we are formally allowed to substitute $\dot{\gamma}_{y z}(t)=\gamma \delta(t)$ in this equation, equivalent to applying a strain step of size $\gamma$ at time zero, and obtain a stress $G(t)$ at later times. This fact gives a meaning to $G(t)$ independent from its definition through oscillatory experiments. We consider this to be the true content of Boltzmann's superposition principle, ${ }^{40}$ which states that the stress is the sum of the responses to delta pulses in the past. We can go even further and derive from this the famous Green-Kubo expression for the shear relaxation modulus $\mathrm{G}(\mathrm{t})=\beta \mathrm{V}\left\langle\sigma_{y z}(\mathrm{t}) \sigma_{y z}(0)\right\rangle$, with $\mathrm{V}$ being the volume of the system, $\beta$ being $\left(k_{\mathrm{B}} \mathrm{T}\right)^{-1}$ with $k_{\mathrm{B}}$ being the Boltzmann constant, and the angular brackets denoting an equilibrium average. Finally, by applying an oscillatory strain and calculating the work performed to keep the oscillation going on, we easily find that the dissipated work is proportional to the loss modulus $G^{\prime \prime}(\omega)=\int_{0}^{\infty} G(t) \omega$ $\cos (\omega t) d t .41,42$

The similarity between Eq. (4) and the equations governing equilibrium rheology has led Vermant et al. ${ }^{2}$ to state that $G_{\perp}^{\prime}$ and $G_{\perp}^{\prime \prime}$ seem to retain their physical meaning of storage and loss modulus. Since the work done during one cycle of the orthogonal probing is proportional to the total stress and the latter is just the sum of the unchanged stress caused by the perturbing flow and the one caused by the probing flow, $G_{\perp}^{\prime \prime}$ indeed must be the additional work to be performed to keep the oscillation going on top of the perturbing flow. We therefore will call $G_{\perp}^{\prime}$ and $G_{\perp}^{\prime \prime}$ the storage and loss moduli. In this paper, we investigate if the other two relationships mentioned above are valid. So we will test if $G_{\perp}(t, \dot{\gamma})$ is equal to the stress measured after applying unit step strain orthogonal to a perturbing flow, and if a generalized Green-Kubo relation still holds.

We want to emphasize that the model of Yamamoto presented here has only served as a framework to clearly state our objectives. The results that we will present only depend on the microscopic particle model, and, in particular, are independent of any rheological model used to describe the results.

The equations for the storage and loss moduli in parallel superposition contain an additional term that depends on the derivative of the spectrum with respect to shear rate. We do not see a way to derive an equation analogous to Eq. (4) in this case. Therefore, we do not have available a parallel oscillatory modulus that can be compared with the parallel step strain modulus. Hence we do not consider PSR in this paper.

\section{SYSTEM AND METHODS}

In this section, we describe the system that we have simulated and some of the methods that we have used to calculate the various properties presented in this paper.

\section{A. System}

As mentioned before, it is our aim in this paper to investigate the consequences of an imposed shear flow on rheological probing techniques orthogonal to that shear flow. We want to restrict ourselves to the influence of the bare shearing flow. Therefore, we have chosen a very simple soft matter system in which the imposed flow has no influence on the internal properties of the particles, nor on their mutual interactions. We will briefly comment on possible changes to our findings caused by the latter in Sec. V. Since, moreover, we did not want to run into specific problems that go with high volume fractions and the glassy state, we have chosen as our system a melt of thirteen armed stars at a pressure of $1 \mathrm{~atm}$. So, the system consists of particles, which interact through an effective potential advocated by Likos and co-workers ${ }^{37}$

$$
\begin{aligned}
\phi\left(r_{i j}\right)= & \left(\frac{5 k_{\mathrm{B}} \mathrm{T}}{18}\right) f^{1.5}\left[-\ln \left(\frac{r_{i j}}{\sigma_{\mathrm{L}}}\right)+\left(1+\frac{\sqrt{f}}{2}\right)^{-1}\right] \quad r_{i j} \leq \sigma_{\mathrm{L}} \\
= & \left(\frac{5 k_{\mathrm{B}} \mathrm{T}}{18}\right) f^{1.5}\left(1+\frac{\sqrt{f}}{2}\right)^{-1}\left(\frac{\sigma_{\mathrm{L}}}{r_{i j}}\right) \\
& \times \exp \left(-\sqrt{f}\left(\frac{r_{i j}-\sigma_{\mathrm{L}}}{2 \sigma_{\mathrm{L}}}\right)\right) \quad r_{i j}>\sigma_{\mathrm{L}},
\end{aligned}
$$

where $r_{i j}$ is the distance between particles $i$ and $j$, i.e., $r_{i j}=\left|\vec{r}_{i}-\vec{r}_{j}\right|$ with $\vec{r}_{i}$ being the position vector of the $i$ th particle. The first line in Eq. (6) describes repulsions at distances smaller than $\sigma_{L}$, while the second line describes the smooth decay of the entropic repulsions to zero at larger distances. $f$ is the functionality of the stars, i.e., their number of arms. In the present application, $f=13 . \sigma_{L}$ sets the length scale of the model.

In order to have some appreciation of the system, in Fig. 1 we present the Likos potential for stars with 13 arms, together

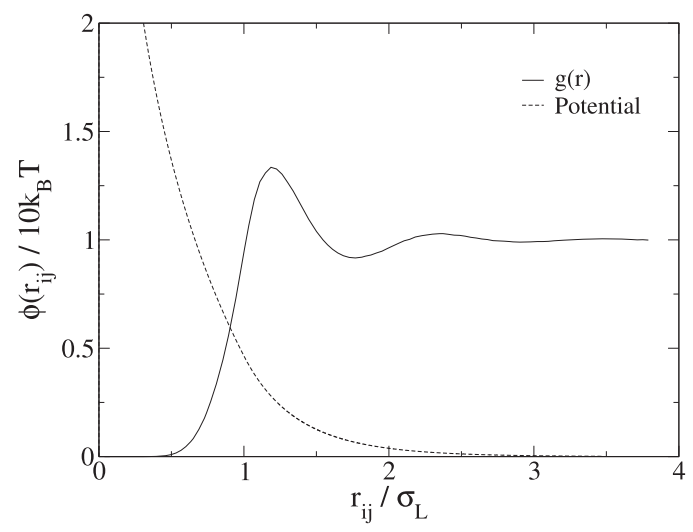

FIG. 1. Potential of mean force as a function of dimensionless inter-star distance for two stars with a functionality of thirteen. The solid line represents the resulting radial distribution function $g(r)$ in a melt. The dotted line is the potential scaled to fit on the graph. The parameter $\sigma_{L}$ is fixed by setting the pressure equal to one atm for the given number density. 
with the radial distribution function for a system with a number density of 0.672 particles per cubic $\sigma_{L}$ and a temperature of $273 \mathrm{~K}$; the potential was set equal to zero at distances beyond $R_{C}=3.48 \sigma_{L}$. Taking the value of $r_{i j}$ where the radial distribution goes through one for the first time as the diameter of the particle, we find a diameter of one $\sigma_{L}$ and next a volume fraction of 0.35. If, on the other hand, we take the value of $r_{i j}$ at the first peak of $g\left(r_{i j}\right)$ to be the diameter, we obtain a diameter of $1.2 \sigma_{\mathrm{L}}$ and a volume fraction of 0.61 . A hard sphere representation of the present model would probably have a diameter and corresponding volume fraction between these two extremes.

In the remaining part of this paper, all quantities will be presented in SI. Therefore, in Table I, we have collected all parameter values in SI, together with some other information defining the simulation. The value of $\sigma_{L}$ was adjusted to obtain a pressure of $1 \mathrm{~atm}$ with the given number density.

\section{B. Orthogonal superposition propagator}

Since we only describe the motion of the centers of mass of the stars using the potential of mean force, this means that we have eliminated all the degrees of freedom that go with the arms from our simulation. In order to obtain the correct dynamics for the centers of mass, we must supply friction forces to them and use a stochastic propagator. With the rather large frictions applying in the present case, we may move forward the particles by the Brownian Dynamics propagator. Forces are derived from the total potential

$$
\Phi\left(r^{3 N}\right)=\sum_{\langle i j\rangle} \phi\left(r_{i j}\right)
$$

The sum $\langle i j\rangle$ runs through all pairs of particles.

In order to implement orthogonal superposition flow as defined in Eq. (1), we make use of the following equations of motion:

$$
\begin{aligned}
d x_{i} & =-\frac{1}{\xi} \frac{\partial \Phi}{\partial r_{i j}} \frac{\partial r_{i j}}{\partial x_{i}} d t+u_{\alpha_{i}, x} d t+f_{i, x} \\
d y_{i} & =-\frac{1}{\xi} \frac{\partial \Phi}{\partial r_{i j}} \frac{\partial r_{i j}}{\partial y_{i}} d t+f_{i, y}, \\
d z_{i} & =-\frac{1}{\xi} \frac{\partial \Phi}{\partial r_{i j}} \frac{\partial r_{i j}}{\partial z_{i}} d t+u_{\alpha_{i}, z} d t+f_{i, z}
\end{aligned}
$$

TABLE I. System parameters.

\begin{tabular}{lc}
\hline \hline Parameter & Value \\
\hline Temperature $\mathrm{T}$ & $273 \mathrm{~K}$ \\
Pressure P & $2.82 \mathrm{~atm}$ \\
Number density $\rho$ & $2.959 \times 10^{24} \mathrm{~m}^{-3}$ \\
Box length $L_{b o x}$ & $70 \mathrm{~nm}$ \\
Number of particles $\mathrm{N}_{\mathrm{t}}$ & 1015 \\
Functionality $f$ & 13 \\
Distance parameter $\sigma_{\mathrm{L}}$ & $6.1 \mathrm{~nm}$ \\
Cutoff radius $\mathrm{R}_{\mathrm{C}}$ & $21.2 \mathrm{~nm}$ \\
Friction coefficient $\xi$ & $8 \times 10^{-3} \mathrm{~kg} \mathrm{~s}^{-1}$ \\
\hline \hline
\end{tabular}

Here $d x_{i}$ is the increment of $x_{i}$ during the time interval of length $d t, \xi$ is a friction coefficient and $f_{i, x}, f_{i, y}$, and $f_{i, z}$ are the components of the Brownian stochastic displacements. The latter obey the fluctuation dissipation theorem

$$
\left\langle f_{i, \alpha} f_{j, \beta}\right\rangle=\frac{2 k_{\mathrm{B}} \mathrm{T}}{\xi} \delta_{\alpha, \beta} \delta_{i, j} d t
$$

where $k_{\mathrm{B}}$ is the Boltzmann constant and $\mathrm{T}$ is the temperature. $\delta_{\alpha, \beta}$ is the Kronecker delta, which is equal to zero unless $\alpha=\beta$, when it is equal to one. The friction coefficient was taken from a previous paper on the same system. ${ }^{43}$

The velocities $u_{\boldsymbol{\alpha}_{i}, x}$ and $u_{\boldsymbol{\alpha}_{i}, z}$ are the average flow velocities in the $\mathrm{x}$ - and $\mathrm{z}$-directions, at the position of particle $i$. In order to measure these velocities, we divide the system into 10 slabs along the $y$-direction and calculate the average velocities $\left\langle\dot{x}_{i}(t)\right\rangle_{\alpha}$ and $\left\langle\dot{z}_{i}(t)\right\rangle_{\alpha}$ in each slab $\alpha$ at each time step. Here $\dot{z}_{i}=d z_{i} / d t$, etc. The flow velocities are then updated for every slab according to

$$
\begin{aligned}
& u_{\alpha, x}(t+d t)=\left\langle\dot{x}_{i}(t)\right\rangle_{\alpha}\left(1-e^{-d t / \tau_{f}}\right)+u_{\alpha, x}(t) e^{-d t / \tau_{f}} \\
& u_{\alpha, z}(t+d t)=\left\langle\dot{z}_{i}(t)\right\rangle_{\alpha}\left(1-e^{-d t / \tau_{f}}\right)+u_{\alpha, z}(t) e^{-d t / \tau_{f}}
\end{aligned}
$$

Here, $\tau_{f}$ characterizes how far in the future, the present average velocities co-determine the flow velocities, in order to smooth the otherwise wildly fluctuating flow velocities. $\tau_{f}$ should be chosen large enough to effectively time-smooth the measured flow fields, but small enough not to influence the physics.

The actual flow field that develops in the system is imposed through Lees-Edwards boundary conditions, which are applied at the two bounding planes of the box perpendicular to the gradient direction. In the present application, the gradient direction is the same for the steady defining flow and for the oscillatory probing flow. In an OSR simulation, i.e., when both flows are present, a particle leaving the box through the upper y-plane comes back into the system through the lower $y$-plane with a displacement in the $\mathrm{x}$-direction of $-\dot{\gamma} \mathrm{Ht}$ (modulo one) and in the $\mathrm{z}$-direction of $-\gamma_{\perp} \sin (\omega t) H ; H$ is the height of the box in the y-direction. Similar operations are applied when a particle leaves the box through the lower $y$-plane and enters through the upper $\mathrm{y}^{-}$ plane, but now with reversed signs. Furthermore, the average velocities in the lowest slab are not measured locally, but are put equal to those in the uppermost slab reduced by $\dot{\gamma} \mathrm{H}$ in the $\mathrm{x}$-direction and by $\gamma_{\perp} \omega \cos (\omega \mathrm{t}) \mathrm{H}$ in the $\mathrm{z}$-direction.

\section{Storage and loss moduli}

As emphasized before, we consider the system as being defined by a shear flow along the $\mathrm{x}$-direction with the gradient along the $y$-direction and being probed by an oscillatory flow in the $\mathrm{z}$-direction with the gradient along the $y$-direction. Unless some nonlinear processes occur in the system, we expect a linear response to the perturbing flow. This means that for sufficiently small values of $\gamma_{\perp}$, Eq. (2) should hold. Here $\mathrm{G}_{\perp}^{\prime}(\omega ; \dot{\gamma})$ is the stress per unit strain amplitude that is in phase 
with the strain, and $\mathrm{G}_{\perp}^{\prime \prime}(\omega ; \dot{\gamma})$ is the stress per unit strain amplitude that is out of phase with the strain. A different way of writing this equation is

$$
\sigma_{y z}(t ; \dot{\gamma})=G_{\perp}^{\prime}(\omega ; \dot{\gamma}) \gamma_{\perp}(\omega, t)+G_{\perp}^{\prime \prime} \frac{1}{\omega} \dot{\gamma}_{\perp}(\omega, t) .
$$

We have calculated the storage and loss moduli by running an OSR simulation as described in Sec. III B, and next fitted the $\sigma_{y z}$ to Eq. (13). In order to obtain a full spectrum, this had to be repeated for different frequencies. Stresses were calculated according to

$$
\sigma_{\alpha \beta}=-\frac{1}{\mathrm{~V}} \sum_{i, j} r_{i j, \alpha} \mathrm{F}_{i j, \beta},
$$

where the sum runs through all pairs, $r_{i j, \alpha}$ is the $\alpha$-component of $\vec{r}_{i}-\vec{r}_{j}$ and $\mathrm{F}_{i j, \beta}$ is the $\beta$-component of the force exerted by particle $j$ on particle $i$.

For a given frequency $\omega$, the time step $d t$ must be chosen small enough to decently sample the oscillating signal, i.e., to have enough points within one cycle. In all runs, we made sure that there were at least 20 points per cycle. On the other hand, we need enough cycles to be able to fit the results to Eq. (13). In all simulations, we have aimed for at least 100 cycles per run. At low frequencies, the stress response is generally noisy, so a larger amplitude $\gamma_{\perp}$ was used to obtain a good signal-to-noise ratio. The maximum value of $\gamma_{\perp}$ was restricted to $5 \%$. At intermediate frequencies (greater than the crossover frequency) the signal was quite smooth and fitting was considerably easier. For the higher shear rates, it is important that the characteristic time $\tau_{f}$ used in Eq. (12) is small enough to not poison the signal. For consistency, we have used $\tau_{f}=d t$ throughout the paper. In Fig. 2, we present three examples of fits of Eq. (13) to the measured stresses. Further results are presented and discussed in Sec. IV A.

\section{Step-strain experiments}

Once the storage and loss moduli have been obtained, $\mathrm{G}_{\perp}(t ; \dot{\gamma})$ can be calculated by Fourier transformation. ${ }^{44}$ As mentioned before, one of our aims in this paper is to investigate if $G_{\perp}(t ; \dot{\gamma})$ is equal to the stress relaxation after a unit step strain perturbation in the direction orthogonal to the steady shear rate, defined as

$$
\mathrm{G}_{\perp}^{\mathrm{s}}(t ; \dot{\gamma})=\lim _{\gamma \rightarrow 0} \frac{1}{\gamma} \sigma_{y z}(t, \gamma ; \dot{\gamma}) .
$$

Here $\gamma$ is the imposed strain step at time zero, and $\sigma_{y z}(t, \gamma ; \dot{\gamma})$ is the resulting stress at later times $t$.

In order to calculate $\sigma_{y z}(t, \gamma ; \dot{\gamma})$, first ten boxes were run for $5 \times 10^{6}$ steps for every shear rate investigated. Next, for every shear rate, each of the ten boxes was run for an additional ten million steps from which 200 frames were stored at regular intervals. So, we had prepared 2000 boxes for every shear rate. Next, for each of the step strain values $\gamma$ investigated, in all boxes all z-coordinates $r_{z, i}$ were replaced by $r_{z, i}$ $+\gamma r_{y, i}$, using periodic boundary conditions of course. Finally all boxes were run for another 10.000 steps, enough for all
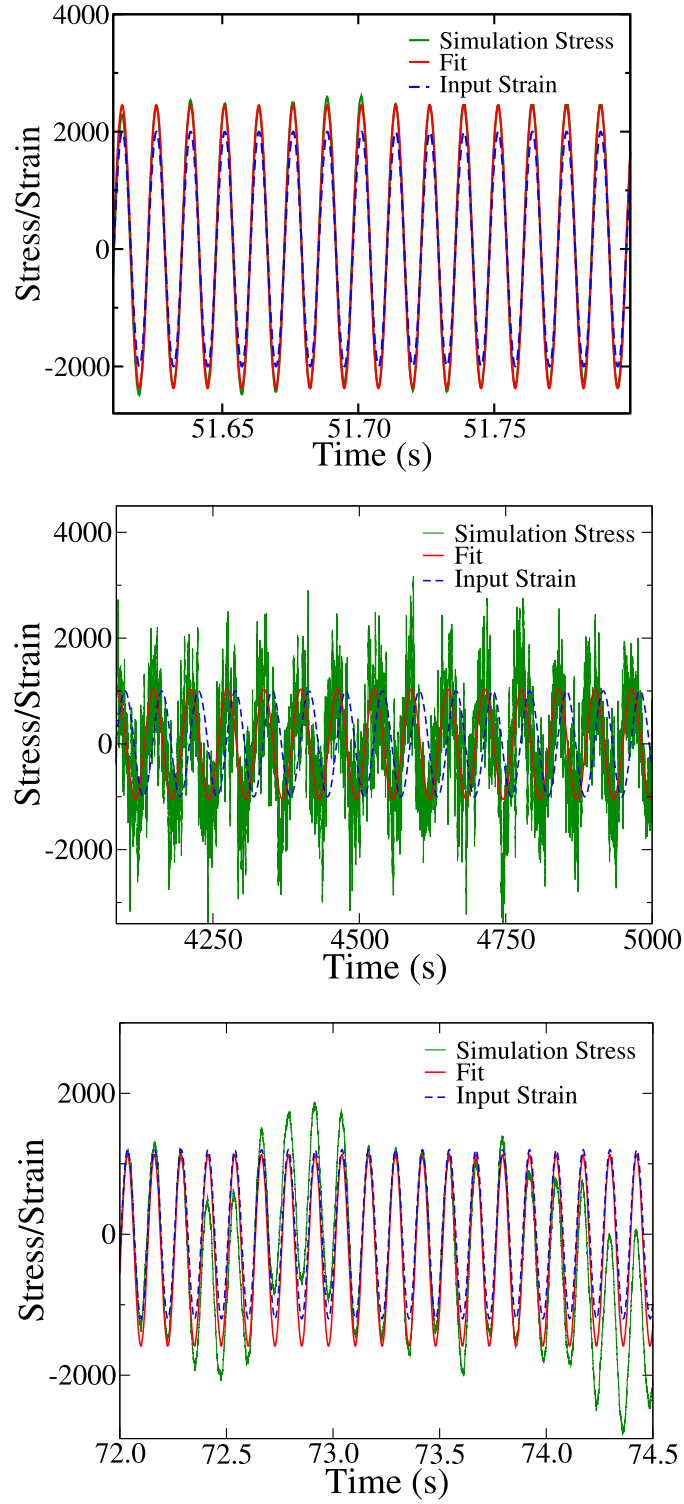

FIG. 2. Sinusoidal fits (red) for the simulated stress (green) after application of different oscillatory perpendicular strains to various steadily sheared boxes. The top panel is for a shear rate of $0.1 \mathrm{~s}^{-1}$ at a frequency of $500 \mathrm{rad} / \mathrm{s}$. The middle panel is for a shear rate of $1 \mathrm{~s}^{-1}$ at a frequency of $0.1 \mathrm{rad} / \mathrm{s}$. The bottom panel is for a shear rate of $2.5 \mathrm{~s}^{-1}$ at a frequency of $50 \mathrm{rad} / \mathrm{s}$. The applied sinusoidal strain, $\gamma_{\perp} \sin (\omega t)$, scaled to become comparable to the stress, is shown in blue. In the top and bottom panels, stresses and strains are almost in phase with each other; in the middle panel, they are out of phase. In the top panel, the difference between the actual values and the fit is hardly visible. In the middle panel, the stress signal is very noisy, but still can be fitted without big problems. The bottom panel shows that with a high shear rate of $2.5 \mathrm{~s}^{-1}$, the fitting is difficult due to a low frequency oscillation that occurs at medium and high frequencies. With even higher shear rates, this poses a severe problem that prevents such systems from being studied.

stresses to relax to zero, and $\sigma_{y z}(t, \gamma ; \dot{\gamma})$ was measured. In all cases, the time step was $0.001 \mathrm{~s}$. 


\section{RESULTS}

In this section, we present the results of our Brownian Dynamics simulations of orthogonal superposition experiments for a system of particles interacting via the Likos potential, and compare them with a variety of results obtained from different calculations.

\section{A. Storage and loss moduli from OSR}

In Fig. 3, we present the $G_{\perp}^{\prime}(\omega ; \dot{\gamma})$ and $G_{\perp}^{\prime \prime}(\omega ; \dot{\gamma})$ obtained from the OSR simulations for different shear rates. The data for $G_{\perp}^{\prime \prime}$ are very noisy at the higher frequencies in all cases. For the lower shear rates, the data for $G_{\perp}^{\prime}$ are somewhat noisy at the lower frequencies. In fact for the non-sheared system, the storage and loss moduli can be better obtained from Fourier transformations of the relaxation modulus. One clearly notices that the moduli of sheared systems increasingly shift to higher frequencies with increasing shear rates. Also a clear decrease of the plateau modulus ( $G_{\perp}^{\prime}$ at high frequencies) with increasing shear rates is evident, indicating an increased fluidization of the sample with increasing steady shear rates.

We want to emphasize that our data in all cases could be well-described by Eq. (13). This implies that the response of the sheared system to the orthogonal probing is linear. In particular, no additional non-linear contributions are needed to describe the data. If we next assume that the orthogonal storage and loss moduli are the sine and cosine transform of a time-domain function, then we can work our way back to Eq. (4). This implies that Eq. (4) holds for an oscillatory shear rate, i.e., for $\dot{\gamma}_{\perp}(t)=\gamma_{\perp} \omega \cos (\omega t)$. This does not mean that Eq. (4) necessarily also holds for other shear rates.

In Fig. 4, we have collected all $\mathrm{G}_{\perp}(\mathrm{t} ; \dot{\gamma})$ obtained by Fourier transformation according to the method of Schwarzl. ${ }^{44}$ Again we notice a decrease of the plateau values $\left(G_{\perp}\right.$ at small times) with increasing steady shear rates. Another interesting characteristic is that the long time behavior of $G_{\perp}$ changes from exponential decay to algebraic decay. As a rough indication, we have fitted an algebraic decay, $G_{\perp}(t ; \dot{\gamma}) \propto t^{-1.5}$, in the case of a shear rate of $2.5 \mathrm{~s}^{-1}$.
Since the storage and loss moduli are the objects that have been obtained directly from the simulation, while the relaxation modulus was obtained from these by a Fourier transform, the former are statistically better defined than the latter. We therefore fitted algebraic expressions to the low frequency part of the storage modulus $G_{\perp}^{\prime}(\omega ; \dot{\gamma}) \propto \omega^{\alpha}$, as shown in Fig. 5. The exponent $\alpha$ depends on the imposed shear rate, as shown in Fig. 6. Clearly $\alpha$ changes from a value of 2 for zero shear rate to 1.5 for an imposed shear rate of $2.5 \mathrm{~s}^{-1}$. The drawn line in this plot, given by $\alpha=2-0.3497 \cdot(\dot{\gamma})^{0.2055}$, only serves as a guide to the eye. Even so, however, the plot seems to indicate that $\dot{\gamma}=0$ is a singular point.

\section{B. Step-strain simulations}

We now discuss the results of our step strain simulations.

\section{Time dependence}

In this subsection, we concentrate on the time dependence of the stress relaxation after a strain step. Therefore all relaxation curves are normalized by their value at time zero. Plateau values will be discussed in Subsection IV B 2.

In Fig. 7 , we present the normalized relaxation function $\sigma_{y z}(t, \gamma ; \dot{\gamma}) / \sigma_{y z}(0, \gamma ; \dot{\gamma})$ for a steady shear rate of $2.5 \mathrm{~s}^{-1}$ and for four values of the strain step $\gamma$ ranging from 0.2 to 0.8 . Surprisingly all curves are identical to within the statistics that we obtained with our procedure and are therefore equal to $G_{\perp}^{s}(t ; \dot{\gamma}) / G_{\perp}^{s}(0 ; \dot{\gamma})$. The same holds for all other steady strain rates that we applied, i.e., normalized relaxation curves for different values of the strain step $\gamma$ only differ by their height. When discussing normalized relaxation curves, we have always averaged over the curves obtained with the four different strain steps.

In Fig. 8, we present the normalized step-strain relaxation curves $G_{\perp}^{s}(t ; \dot{\gamma})$ for all different values of the steady shear rates that we have studied, together with the corresponding normalized $\mathrm{G}_{\perp}(t ; \dot{\gamma})$. It is clearly seen that the change of the characteristic decay times with increasing shear rates are very similar for both cases, but the long time decays develop differently. For zero shear, $G_{\perp}(t ; 0)=G_{\perp}^{s}(t ; 0)$ to a good

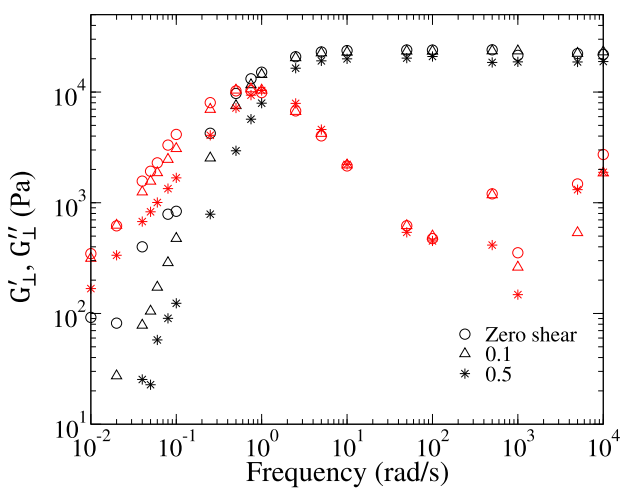

(a)

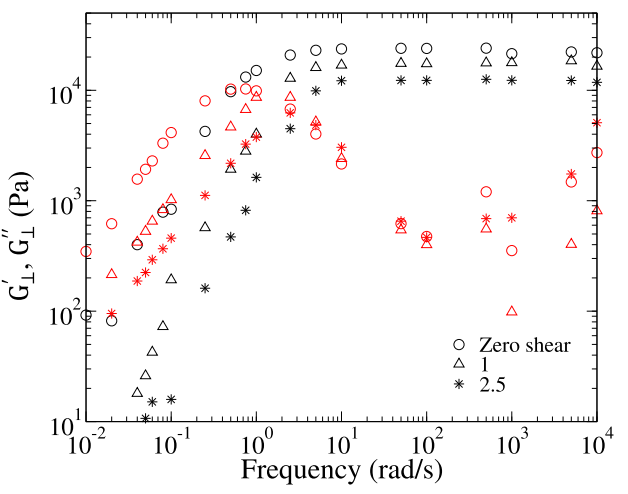

(b)
FIG. 3. Storage and loss moduli for steadily sheared systems. $\mathrm{G}_{\perp}^{\prime}(\omega ; \dot{\gamma})$ is shown in black and the $G_{\perp}^{\prime \prime}(\omega ; \dot{\gamma})$ in red. (a) Steady shear rates of $0.0 \mathrm{~s}^{-1}$ (circles), $0.1 \mathrm{~s}^{-1}$ (triangles), and 0.5 $\mathrm{s}^{-1}$ (asterisks). While the curves of 0.1 $\mathrm{s}^{-1}$ are almost indistinguishable from the zero shear response, those of $0.5 \mathrm{~s}^{-1}$ are slightly shifted to higher frequencies. A slight decrease of plateau modulus for $0.5 \mathrm{~s}^{-1}$ can be seen. (b) Steady shear rates of $0.0 \mathrm{~s}^{-1}$ (circles), $1.0 \mathrm{~s}^{-1}$ (triangles), and $2.5 \mathrm{~s}^{-1}$ (asterisks). Shifts along the frequency axis and decreases of plateau values are more pronounced. 


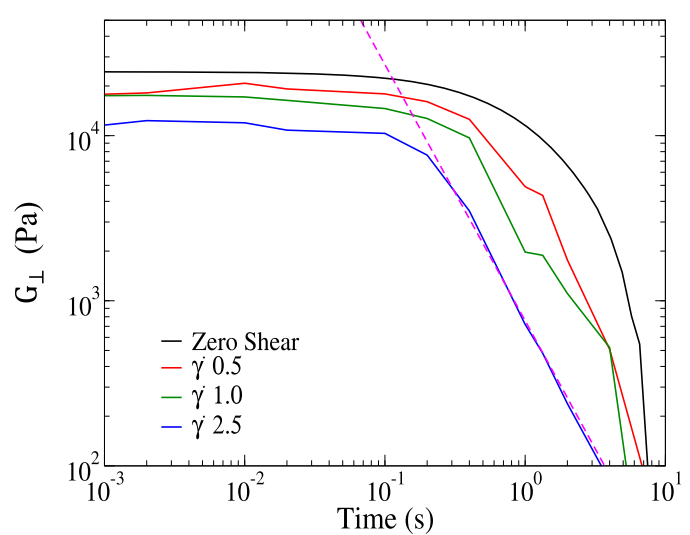

FIG. 4. $\mathrm{G}_{\perp}(t ; \dot{\gamma})$ for different shear rates obtained by the method of Schwarzl applied to the OSR moduli shown in Fig. 3. The plateau moduli decrease and the terminal decay becomes algebraic with increasing shear. For the largest shear rate, the terminal algebraic decay is shown by a dotted line with a slope of 1.5 .

approximation. With increasing shear rate, $G_{\perp}^{\text {s }}(t ; \dot{\gamma})$ continues to decay exponentially, whereas $G_{\perp}(t ; \dot{\gamma})$ gradually develops an algebraic tail as discussed in Sec. IV A.

One of the differences between the two experiments is that the displacements in the z-direction are not strictly linear with height y in the oscillatory simulation, whereas they are linear by definition in the initial frames of the step strain simulations. This is a result of the use of a "boundary driven method" in the OSR simulations, in which the linear profile has to develop by itself. We therefore ran a simulation in which the flow fields are applied more directly. This we did by replacing $u_{\alpha_{i}, x}$ by $\dot{\gamma} y_{i}$ and $u_{\alpha_{i}, z}$ by $\omega \gamma_{\perp} \cos (\omega t) y_{i}$ in the propagator Eq. (10). We call this method the "force driven method." In Fig. 9, we have plotted the storage and loss moduli for the

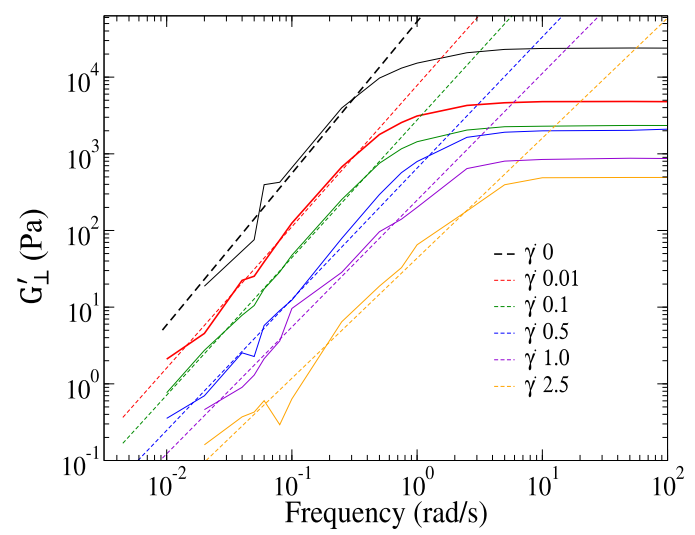

FIG. 5. The figure shows the low frequency part of the storage moduli $\mathrm{G}_{\perp}^{\prime}(\omega ; \dot{\gamma})$ fitted with dashed lines for different shear rates in order to obtain the terminal slope $\alpha$. For visual reasons, the moduli have been shifted vertically with respect to the zero-shear curve (black) by dividing the original $\mathrm{G}_{\perp}^{\prime}(\omega ; \dot{\gamma})$ by a factor of 5,10 , 10,20 , and $25 \mathrm{~Pa}$ for $\dot{\gamma}=0.01,0.1,0.5,1.0$, and $2.5 \mathrm{~s}^{-1}$, respectively, in order to clearly separate them.

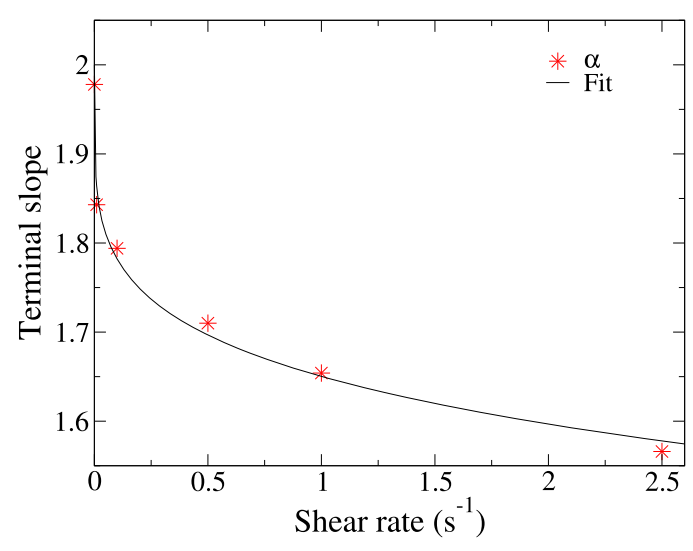

FIG. 6 . The slopes $\alpha$ obtained in 5 shown as a function of shear rate $\dot{\gamma}$. The values range from a maximum of 1.978 for $\dot{\gamma}=0-1.566$ for $\dot{\gamma}=2.5$. The drawn-line fit is given by $\alpha=2-0.3497 \cdot(\dot{\gamma})^{0.2055}$.

case with $\dot{\gamma}=2.5 \mathrm{~s}^{-1}$, both for the boundary driven case and for the force driven method, together with those obtained from the step strain simulation. To within the statistics of our results, one may say that the difference between boundary driven results and force driven results is smaller than the differences between both of them and the step strain results.

We conclude that, for stationary sheared systems, the relaxation spectrum probed by small oscillatory perturbations is different from that obtained with step strain simulations.

\section{Plateau values}

We now consider the time zero values of the relaxation moduli, i.e., the normalization constants $\sigma_{y z}(0, \gamma ; \dot{\gamma})$ used in Subsection IV B 1. For ease of notation, we define $\sigma_{\perp}(\gamma ; \dot{\gamma})=\sigma_{y z}(0, \gamma ; \dot{\gamma})$ and refer to them as peak values.

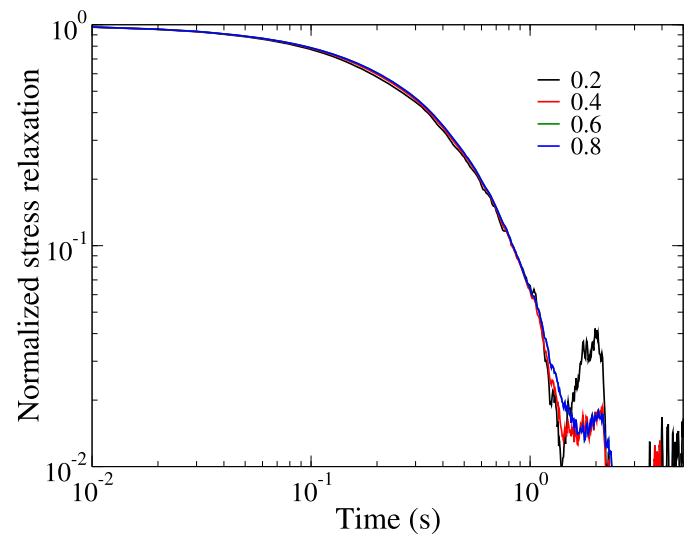

FIG. 7. Normalized stress relaxation functions $\sigma_{y z}(t, \gamma ; \dot{\gamma}) / \sigma_{y z}(0, \gamma ; \dot{\gamma})$ for $\dot{\gamma}=$ $2.5 \mathrm{~s}^{-1}$, after step-strains of $0.2,0.4,0.6$, and 0.8 , all of which collapse onto the same master curve. The behavior is similar for all other shear rates considered. 


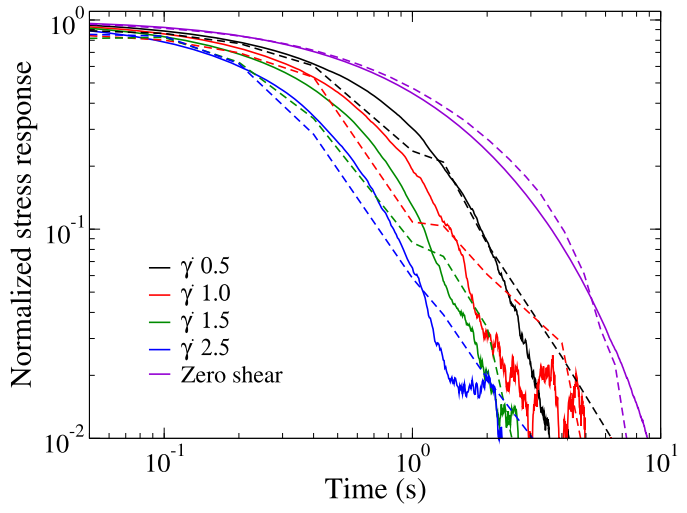

FIG. 8. The average normalized step-strain relaxation curves $G_{\perp}^{\mathrm{s}}(t ; \dot{\gamma})$ (solid lines) for all steady shear rates along with the normalized $\mathrm{G}_{\perp}(\mathrm{t} ; \dot{\gamma})$ (dashed lines). With increasing shear rate, $G_{\perp}^{\mathrm{s}}(t ; \dot{\gamma})$ continues to decay exponentially, whereas $\mathrm{G}_{\perp}(\mathrm{t} ; \dot{\gamma})$ gradually develops an algebraic tail.

Since the stress immediately after a strain step is a nondynamical property, we can easily derive theoretical expressions for it. In Appendix A, we will show that we may write

$$
\sigma_{\perp}(\gamma ; \dot{\gamma})=\sigma_{\perp}^{(1)}(\dot{\gamma}) \gamma+\frac{\sigma_{\perp}^{(3)}(\dot{\gamma})}{6} \gamma^{3}+\ldots
$$

with

$$
\sigma_{\perp}^{(1)}(\dot{\gamma})=\frac{1}{V} \sum_{i<j}\left\langle\left(z_{i j}^{2} \frac{\phi^{\prime \prime}}{r_{i j}^{2}} y_{i j}^{2}-z_{i j}^{2} \frac{\phi^{\prime}}{r_{i j}^{3}} y_{i j}^{2}+\frac{\phi^{\prime}}{r_{i j}} y_{i j}^{2}\right)\right\rangle_{\dot{\gamma}}
$$

and a similar expression for $\sigma_{\perp}^{(3)}(\dot{\gamma})$. The primes in Eq. (17) denote differentiations and $x_{i j}=x_{i}-x_{j}$, etc. The angular brackets \langle\rangle$_{\dot{\gamma}}$ denote an average over the configurations sampled from a steadily sheared run. For obvious reasons, terms with even powers of $\gamma$ are absent (see Appendix A). Comparison of

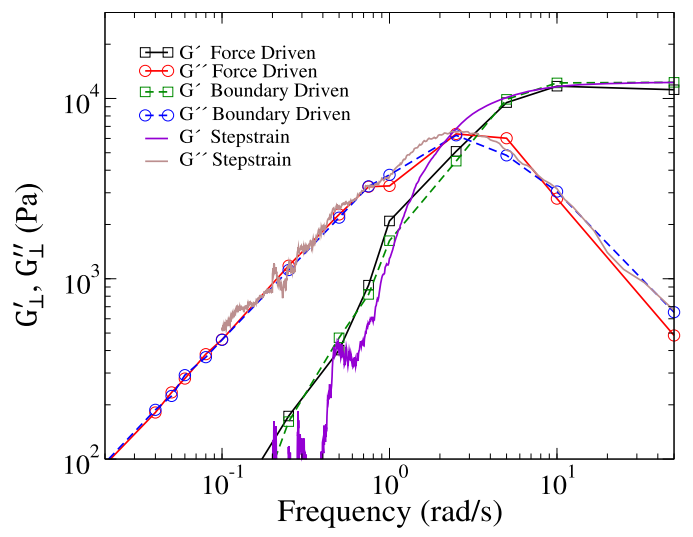

FIG. 9. The relaxation moduli obtained for a shear rate of $2.5 \mathrm{~s}^{-1}$ for both boundary driven and force-driven methods (symbols) and the step strain (solid line with no symbols). The results from both methods are in good agreement and differ significantly from those obtained with the step strain simulations. This shows that the onset of power-law behavior with increasing shear rates at low frequencies is not a consequence of the implementation method.
Eqs. (15) and (17) shows that $G_{\perp}^{s}(0 ; \dot{\gamma})=\sigma_{\perp}^{(1)}(\dot{\gamma})$. We refer to $\sigma_{\perp}^{(1)}(\dot{\gamma})$ as the step strain plateau value since it is equal to the constant value to which the storage modulus corresponding to $G_{\perp}^{s}(t ; \dot{\gamma})$ converges for large frequencies.

In Fig. 10, we present $\sigma_{\perp}(\gamma ; \dot{\gamma}) / \gamma$ (symbols) for various values of the strain step and compare them with the theoretical results (dashed lines). The figure clearly confirms the theory. Remaining small differences could be a result of finite size effects which are different for the simulated plateau values and the calculated ones.

In Figs. 11(a) and 11(b), we present $\sigma_{\perp}^{(1)}(\dot{\gamma})$ (circles) and $\sigma_{\perp}^{(3)}(\dot{\gamma})$ (circles) as a function of the shear rate of the imposed stationary flow. Included in Fig. 11(a) are also $\mathrm{G}_{\perp}(0 ; \dot{\gamma})$ (triangles) obtained from the oscillatory simulations. These values clearly agree with those from the step strain simulations. So, although moduli obtained from oscillatory experiments do not agree with those obtained from step strain experiments in their long time behavior, they do have the same short time values.

Now, let us shortly discuss what information is to be found in $\sigma^{(1)}(\dot{\gamma})$ and $\sigma^{(3)}(\dot{\gamma})$. From Eq. (17), we notice that $\sigma^{(1)}(\dot{\gamma})$ is a sum over pair-terms, each averaged over the configurations obtained from a sheared simulation. As such it may be considered to be a way of probing the perturbation of the pair distribution function by the applied stationary flow. We therefore write Eq. (17) as

$$
\sigma_{\perp}^{(1)}(\dot{\gamma})=\frac{\rho^{2}}{2} \int d^{3} r g(\vec{r} ; \dot{\gamma})\left(z^{2} \frac{\phi^{\prime \prime}}{r^{2}} y^{2}-z^{2} \frac{\phi^{\prime}}{r^{3}} y^{2}+\frac{\phi^{\prime}}{r} y^{2}\right)
$$

where $g(\vec{r} ; \dot{\gamma})$ is the perturbed pair distribution function. Note that we have assumed a homogeneous and isotropic density of particles $\rho$. A similar equation holds for $\sigma_{\perp}^{(3)}(\dot{\gamma})$. For non-zero shear rates, we write approximately

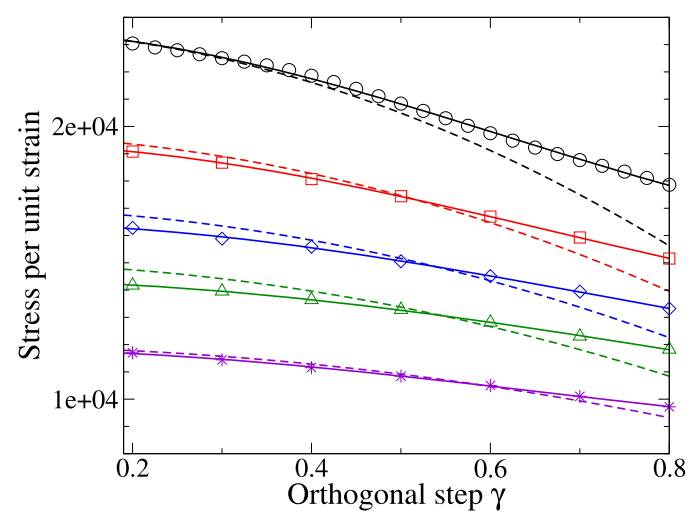

FIG. 10. Stress per unit step-strain, $\sigma_{\perp}(\gamma ; \dot{\gamma}) / \gamma$, for different values of the step magnitude $\gamma$ (symbols) and the theoretical fits for them shown by dashed lines. The curves from top to bottom are for shear rates $0,0.5,1.0,1.5$, and 2.5 , respectively. The drawn lines through the symbols are guides to the eye. Close agreement with theory is clearly seen. 


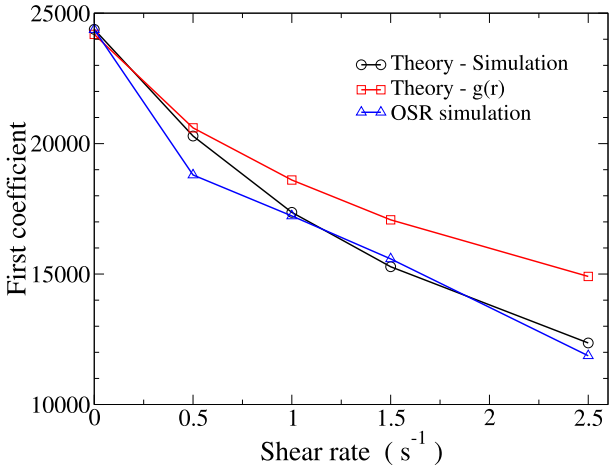

(a)

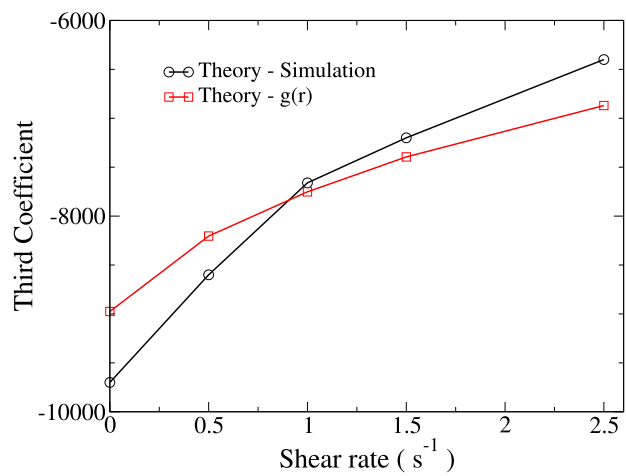

(b)

FIG. 11. Panel (a) shows the first coefficient $\sigma_{\perp}^{(1)}(\dot{\gamma})$ as a function of shear rate calculated by two different methods: simulation of expression ${ }^{17}$ (circles) and by using the spherical part of the radial distribution function $g^{(0)}(r ; \dot{\gamma})$ (squares). ${ }^{18}$ These are compared with the plateau modulus from OSR (triangles). For all shear rates, the coefficient calculated from simulation is very close to the plateau values. When using $g^{(0)}(r ; \dot{\gamma})$, the first coefficient differs substantially from OSR plateau values at large imposed shear rates, while at smaller shear rates both results converge to each other. Panel (b) shows the third coefficient calculated from the expression and from $g^{(0)}(r ; \dot{\gamma})$.

$$
\begin{aligned}
g(\vec{r} ; \dot{\gamma})= & g^{(0)}(r ; \dot{\gamma})+g^{(1)}(r ; \dot{\gamma}) \frac{x y}{r^{2}} \dot{\gamma} \\
& +\left[g^{(2,1)}(r ; \dot{\gamma}) \frac{x^{2} y^{2}}{r^{4}}+g^{(2,2)}(r ; \dot{\gamma}) \frac{x^{2}+y^{2}}{r^{2}}\right] \dot{\gamma}^{2}+\ldots
\end{aligned}
$$

In this equation, we have accounted for the symmetry imposed by the shear flow. The coefficients $g^{(0)}(r ; \dot{\gamma})$, etc., may still depend on $\dot{\gamma}$. In particular, we leave open if they are analytic or singular functions of the shear rate. In Fig. 12, we depict the spherical part $g^{(0)}(r ; \dot{\gamma})$ of the perturbed pair function. It is clearly seen that also the spherical part of the distribution function is severely changed by the stationary shear flow. Restricting ourselves to just this contribution to the pair function, we obtained the results denoted " $g(r)$ " (squares) in Figs. 11(a) and 11(b). For shear rates below $0.5 \mathrm{~s}^{-1}$, the $\sigma_{\perp}^{(1)}(\dot{\gamma})$ within this approximation are rather close to the actual

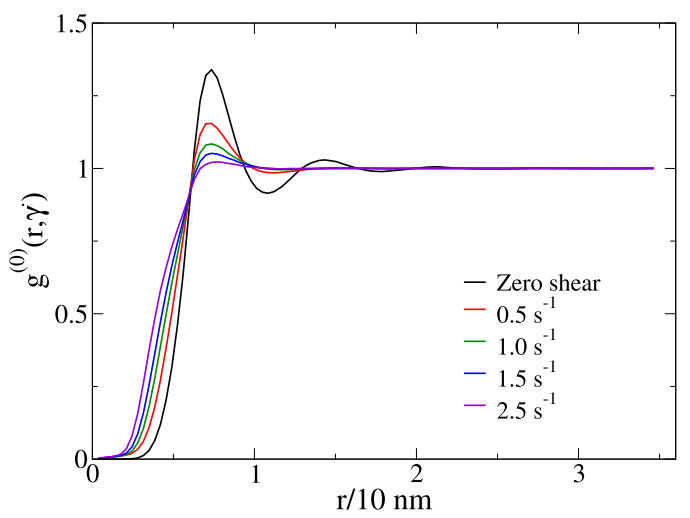

FIG. 12. The spherical part of the radial distribution function, $g^{(0)}(r ; \dot{\gamma})$, for different shear rates. The first peak reduces in magnitude, showing the effect of increasing shear on removing correlations between particle positions. values. At larger shear rates, the difference between the actual coefficients and those obtained with the spherical pair function indicates that the contribution of the non-spherical terms must be substantial. Including the next term of the pair distribution function, the one proportional to $\dot{\gamma}$ will make no difference. This can be inferred from the fact that including this term gives rise to an additional sum of terms in the integrand which are all proportional to odd powers of $x$ and $y$, which will all become zero after an integration over all space. A similar argument shows that the next terms, proportional to $\dot{\gamma}^{2}$, do contribute to the integrals. Since possible density gradients may further complicate the picture, we do not investigate this here, referring for a more detailed discussion to Ref. 45. A phenomenological analysis of the role of configurations, based on the Giesekus model, has been presented in Ref. 46.

Before ending this section, let us conclude from the results in this section and those of Sec. IV B 1 that for the sheared systems, relaxation moduli obtained from oscillatory simulations are largely equal to those obtained from step strain simulations, but that their moduli differ in late time behavior. The strong version of the Boltzmann principle, as we defined it in the Introduction, is therefore not valid. It even seems that in this respect, the zero shear case is a singular case, as strongly suggested by the results in Fig. 6 .

\section{Violation of Green-Kubo}

Under zero-shear conditions, the shear relaxation modulus is proportional to the time correlation function of any offdiagonal component of the stress tensor. Naively, one might argue that the imposed stationary shear flow serves only to define the system and that the Green-Kubo relations should still hold, with the exception that time correlation functions should be calculated in the sheared ensemble. We therefore ask whether $\mathrm{G}_{\perp}(t ; \dot{\gamma})$ given by 


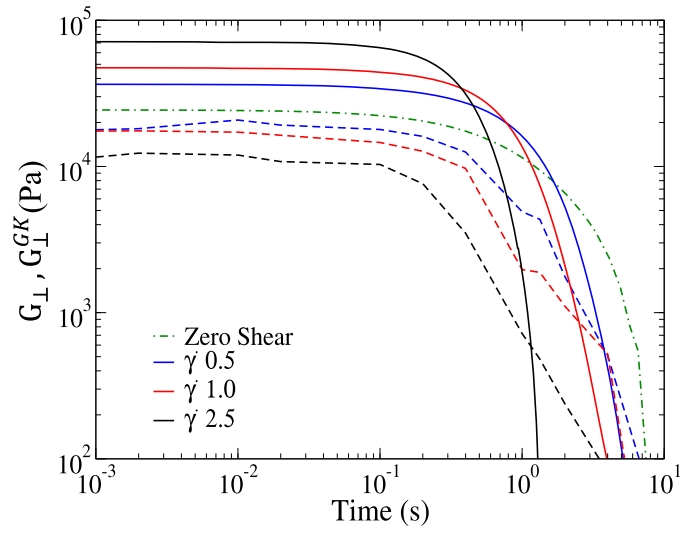

FIG. 13. Shear relaxation moduli from the Green-Kubo relation, Eq. (20), $G_{\perp}^{G K}(t ; \dot{\gamma})$ (solid lines), for three shear rates of $0.5,1.0$, and $2.5 \mathrm{~s}^{-1}$. Moduli calculated from OSR results, $G_{\perp}(t ; \dot{\gamma})$, are shown as dashed lines. The zero-shear relaxation modulus is shown as a dashed-dotted line for reference. Clearly the GK moduli are very different from the "real" moduli $G_{\perp}(t ; \dot{\gamma})$. Not only do they have a higher plateau value which, contrary to those of $G_{\perp}(t ; \dot{\gamma})$, increases with shear rate, but they also decay much later than their counterparts and in a qualitatively different manner. Clearly the Green-Kubo relations do not hold under large shear rates.

$$
\mathrm{G}_{\perp}^{\mathrm{GK}}(\mathrm{t} ; \dot{\gamma})=\frac{\mathrm{V}}{k_{\mathrm{B}} \mathrm{T}}\left\langle\sigma_{y z}(\mathrm{t}) \sigma_{y z}(0)\right\rangle_{\dot{\gamma}}
$$

On the other hand, one of the easiest ways to derive the Green-Kubo relations at zero shear is through the analysis of step strain experiments, which for completeness we show in Appendix B. Now that we have seen that under shear, orthogonal step strain and oscillatory experiments yield different relaxation moduli, one may expect that Green-Kubo also does not apply.

In Fig. 13, we present $G_{\perp}^{\mathrm{GK}}(\mathrm{t} ; \dot{\gamma})$ calculated using Eq. (20). In order to obtain good statistics, 30 pre-equilibrated boxes were run for $10 \times 10^{6}$ steps each, and the stress correlations from them were averaged to yield the results. It is clearly seen in Fig. 13 that the Green-Kubo moduli do not agree with the OSR moduli. While the initial values are decreasing for the OSR results, they are increasing for the GK results. The dynamics is somewhat similar in the sense that both moduli decay earlier with increasing shear rates, but the GK moduli do not develop the characteristic algebraic tails as the OSR moduli do. We checked that the normalised GK moduli are also different from the normalized step strain moduli. Only in the non-sheared system do all moduli, i.e., the OSR moduli, the GK moduli, and the step strain moduli, agree.

\section{SUMMARY AND DISCUSSION}

We have investigated, by means of Brownian dynamics simulations, the applicability of Boltzmann's superposition principle to orthogonal superposition rheology. We prepared our systems by shearing them along the $\mathrm{x}$-axis, with the gradient along the $y$-axis, and maintained this shear during all subsequent "experiments." We investigated these systems by measuring the response to the application of small probing perturbations orthogonal to the applied steady shear. The probing perturbations were either a small step strain perturbation or an oscillating perturbation, both in the $\mathrm{z}$-direction with the gradient along the $y$-axis. These perturbations give rise to the following responses:

$$
\begin{aligned}
& \sigma_{y z}=\gamma G_{\perp}^{s}(t ; \dot{\gamma}), \\
& \sigma_{y z}=\int_{-\infty}^{t} G_{\perp}\left(t-t^{\prime} ; \dot{\gamma}\right) \dot{\gamma}_{\perp}\left(t^{\prime}\right) d t^{\prime},
\end{aligned}
$$

with $\gamma$ being the value of the strain step and $\dot{\gamma}_{\perp}(t)$ the time dependent orthogonal shear rate. Both shear moduli $G_{\perp}^{\mathrm{s}}$ and $\mathrm{G}_{\perp}$ depend parametrically on the applied steady shear rate $\dot{\gamma}$. At zero shear rate, i.e., when $\dot{\gamma}=0$, both shear moduli are equal. We have found in this paper that this is not true anymore for non-zero shear rates.

Although the initial values of both moduli are the same, and their decay times are roughly the same, their late time dependencies are different. While $G_{\perp}$ develops an algebraic tail with increasing steady shear rates, the step strain modulus $G_{\perp}^{s}$ does not do so. We quantified these findings by calculating the exponents $\alpha$ of the low frequency algebraic increase of the storage modulus, $G_{\perp}^{\prime}(\omega ; \dot{\gamma}) \propto \omega^{\alpha}$, as a function of the imposed steady shear rate. It turns out that $\alpha$ continuously approaches its zero shear rate value of 2 , when shear rates become very small, but the corresponding curve is singular, i.e., has no derivative, at zero shear rate. This is an indication that even at the smallest shear rates, orthogonal probing cannot be considered to be a linear probing of a linearly perturbed system. Apparently the different "symmetries" of a small steady shear and an oscillatory orthogonal shear with small amplitude do not just give rise to a stress response whose components can be calculated from equilibrium properties. Obviously, combining two steady shear flows can be turned into a simple shear flow by rotating the coordinate system. It will be interesting to investigate the same problem for two oscillatory flows.

Besides their differences, $G_{\perp}^{\mathrm{s}}$ and $G_{\perp}$ also have similarities. Both of them accelerate, i.e., decay at increasingly smaller times with increasing shear rates, to roughly the same extent. For the step-strain experiments, this may be a result of shear induced diffusion. ${ }^{47}$ When an oscillatory flow is applied orthogonal to the imposed shear flow, the shear induced diffusion processes are severely influenced, probably giving rise to additional diffusion modes. The latter may be the cause of the algebraic decay.

As mentioned in the main text, the initial values $G_{\perp}^{s}(0 ; \dot{\gamma})$ and $G_{\perp}(0 ; \dot{\gamma})$ do coincide. We have presented a simple equation that allows to calculate the dependence of these quantities on the imposed steady shear rate. It is interesting that, for small imposed shear rates, the numerical evaluation of this equation only requires the spherical part of the pair correlation function as a function of the imposed shear rate. This means that the vertical shift factor in a time-shear rate superposition presentation, analogous to a time-temperature superposition 
presentation, of the data can be simply related to the perturbation of the spherical part of the pair correlation function caused by an imposed steady shear flow. For interesting phenomenological relations concerning this type of superposition, see Ref. 48.

Let us finish with a few comments concerning the molecular model that we have used in this paper. We have explicitly not taken into account many aspects that are characteristic for soft matter systems. Near equilibrium, usually a wide range of time scales related to internal dynamics is visible in rheological spectra, among which are Rouse and reptation dynamics in general, or arm retraction moves as in star polymers. ${ }^{49,50}$ With larger shear rates, on a very coarse level, particles may be considered to interact through mean forces augmented with transient forces. ${ }^{51,52}$ As it has been used so far, that model will be insufficient in many cases since it does not take into account the deformation of the individual particles caused by the imposed shear flow. A consequence of this would be that the potential interactions become anisotropic and that the frictions in Eq. (10) should be different for motions in different directions. ${ }^{53}$ It is difficult to predict what the influence of these responses of the particles to the imposed shear flow would be, but one may speculate that they help to delay the onset of the algebraic tails found in this paper to larger shear rates. This seems to be corroborated by results presented in Ref. 10.

\section{ACKNOWLEDGMENTS}

The authors would like to thank George Petekidis from FORTH, Crete, for introducing us to the subject. The work leading to these results has received funding from the People Programme (Marie Skłodowska-Curie Actions) of the European Union's Seventh Framework Programme (No. FP7/20072013) under REA Grant Agreement No. 607937-SUPOLEN project.

\section{APPENDIX A: TAYLOR EXPANSION OF THE VIRIAL STRESS AFTER A STRAIN STEP}

In this Appendix, we will show that the virial stress $\sigma_{\perp}(\gamma ; \dot{\gamma})$ at time $t=0^{+}$after a strain step may be written as a power expansion in $\gamma$ of the form

$$
\sigma_{\perp}(\gamma ; \dot{\gamma})=\sum_{n=0}^{\infty} \frac{\sigma_{\perp}^{(n)}(\dot{\gamma})}{n !} \gamma^{n}
$$

Since the stress must change sign when $\gamma$ changes sign, only odd powers of $\gamma$ will have non-zero coefficients.

To determine the coefficients $\sigma_{\perp}^{(n)}(\dot{\gamma})$, we simply mimic the step strain experiment, by sampling from an ensemble of systems applicable at the given shear rate $\dot{\gamma}$, performing a strain step of size $\gamma$, and measuring the stress immediately after the step. Since the strain step changes $\vec{r}_{i}$ into $\vec{r}_{i}+\gamma y_{i} \widehat{e}_{z}$, the relevant stress component may be written as

$$
\sigma_{\perp}(\gamma ; \dot{\gamma})=\frac{1}{\mathrm{~V}} \sum_{i<j}\left\langle y_{i j} \frac{\phi^{\prime}\left(r_{i j}+\Delta_{i j}\right)}{r_{i j}+\Delta_{i j}}\left(z_{i j}+\gamma y_{i j}\right)\right\rangle_{\dot{\gamma}} .
$$

In this equation, we have used the virial expression for stress given in Eq. (14), while the angular brackets \langle\rangle$_{\dot{\gamma}}$ indicate an average over the ensemble applicable when the stationary shear rate equals $\dot{\gamma}$. The difference $\Delta_{i j}$ is given by

$$
\Delta_{i j}=\frac{z_{i j} y_{i j}}{r_{i j}} \gamma+\frac{1}{2}\left(\frac{y_{i j}^{2}}{r_{i j}}-\frac{z_{i j}^{2} y_{i j}^{2}}{r_{i j}^{3}}\right) \gamma^{2}+\frac{1}{2}\left(\frac{z_{i j}^{3} y_{i j}^{3}}{r_{i j}^{5}}-\frac{z_{i j} y_{i j}^{3}}{r_{i j}^{3}}\right) \gamma^{3},
$$

where we have expanded upto the third order in $\gamma$. The second and third powers of $\Delta_{i j}$ are given by

$$
\begin{aligned}
\Delta_{i j}^{2} & =\frac{z_{i j}^{2} y_{i j}^{2}}{r_{i j}^{2}} \gamma^{2}+\left(\frac{z_{i j} y_{i j}^{3}}{r_{i j}^{2}}-\frac{z_{i j}^{3} y_{i j}^{3}}{r_{i j}^{4}}\right) \gamma^{3}, \\
\Delta_{i j}^{3} & =\frac{z_{i j}^{3} y_{i j}^{3}}{r_{i j}^{3}} \gamma^{3},
\end{aligned}
$$

where again we have retained only terms up to the third order in $\gamma$.

Expanding the pair interaction potential to third order in $\Delta_{i j}$, we find

$$
\begin{aligned}
\sigma_{\perp}(\gamma ; \dot{\gamma})= & \frac{1}{\mathrm{~V}} \sum_{i<j}\left\langle\left(\phi^{\prime}+\phi^{\prime \prime} \Delta_{i j}+\frac{\phi^{\prime \prime \prime}}{2} \Delta_{i j}^{2}+\frac{\phi^{\prime \prime \prime \prime}}{6} \Delta_{i j}^{3}\right)\right. \\
& \left.\times\left(1+\frac{\Delta_{i j}}{r_{i j}}\right)^{-1}\left(\frac{z_{i j} y_{i j}}{r_{i j}}+\frac{\gamma y_{i j}^{2}}{r_{i j}}\right)\right\rangle_{\dot{\gamma}} .
\end{aligned}
$$

The number of primes with the potential $\phi$ represents the order of the derivative. Expanding the middle factor and next all powers of $\Delta_{i j}$, we obtain the following expressions:

$$
\sigma_{\perp}^{(1)}(\dot{\gamma})=\frac{1}{\mathrm{~V}} \sum_{i<j}\left\langle\left(\frac{\phi^{\prime \prime} z_{i j}^{2} y_{i j}^{2}}{r_{i j}^{2}}-\frac{\phi^{\prime} z_{i j}^{2} y_{i j}^{2}}{r_{i j}^{3}}+\frac{\phi^{\prime} y_{i j}^{2}}{r_{i j}}\right)\right\rangle_{\dot{\gamma}},
$$

$$
\begin{aligned}
\sigma_{\perp}^{(3)}(\dot{\gamma})= & \frac{1}{\mathrm{~V}} \sum_{i<j}\left\langle\left(\frac{z_{i j}^{4} y_{i j}^{4}}{r_{i j}^{4}} \phi^{\prime \prime \prime \prime}+\frac{6 z_{i j}^{2} y_{i j}^{4}}{r_{i j}^{3}} \phi^{\prime \prime \prime}-\frac{6 z_{i j}^{4} y_{i j}^{4}}{r_{i j}^{5}} \phi^{\prime \prime \prime}\right)\right\rangle_{\dot{\gamma}} \\
& +\frac{1}{\mathrm{~V}} \sum_{i<j}\left\langle\left(\frac{15 z_{i j}^{4} y_{i j}^{4}}{r_{i j}^{6}} \phi^{\prime \prime}-\frac{18 z_{i j}^{2} y_{i j}^{4}}{r_{i j}^{4}} \phi^{\prime \prime}+\frac{3 y_{i j}^{4}}{r_{i j}^{2}} \phi^{\prime \prime}\right)\right\rangle_{\dot{\gamma}} \\
& +\frac{1}{\mathrm{~V}} \sum_{i<j}\left\langle\left(\frac{18 z_{i j}^{2} y_{i j}^{4}}{r_{i j}^{5}} \phi^{\prime}-\frac{15 z_{i j}^{4} y_{i j}^{4}}{r_{i j}^{7}} \phi^{\prime}-\frac{3 y_{i j}^{4}}{r_{i j}^{3}} \phi^{\prime}\right)\right\rangle_{\dot{\gamma}} .
\end{aligned}
$$

Both coefficients are averages over configurations of a sheared ensemble of sums of terms, each of which is proportional to even powers of $y_{i j}$ and $z_{i j}$.

Coefficients with even superscripts are averages over configurations of a sheared ensemble of sums of terms, each of which is proportional to odd powers of $y_{i j}$ and $z_{i j}$. Replacing 
the averaging $1 / \mathrm{V} \sum_{i<j}\langle\rangle_{\dot{\gamma}}$ by $\rho^{2} / 2 \int d^{3} \mathrm{rg}(\vec{r} ; \dot{\gamma})$ and noticing that $g(\vec{r} ; \dot{\gamma})$ contains only terms proportional to powers of $x$ and $y$, we find that all terms are proportional to an odd power of $z$ and will become zero after integration over all space. This constitutes a mathematical proof of the physically obvious fact that terms proportional to even powers of $\gamma$ do not contribute in Eq. (A1).

The remaining non-zero terms are all of the type

$$
\int d r F(r) \oint d S z^{\alpha} y^{\beta}=\mathrm{I}_{\alpha, \beta} \int d r \mathrm{~F}(r)
$$

where $\oint d S$ denotes an integral over the unit sphere. The only integrals $\mathrm{I}_{\alpha, \beta}$ that we needed are $\mathrm{I}_{2,0}=4 \pi / 3, \mathrm{I}_{2,2}=4 \pi / 15, \mathrm{I}_{4,0}=$ $4 \pi / 5, \mathrm{I}_{4,2}=4 \pi / 35$, and $\mathrm{I}_{4,4}=4 \pi / 105$. The remaining integrals over $r$ were done numerically.

\section{APPENDIX B: DERIVATION OF GREEN-KUBO EQUATION FOR EQUILIBRIUM SYSTEMS}

In this Appendix, we give a quick derivation of the GreenKubo expression to calculate the stress relaxation after a strain step. The distribution of configurations $r^{3 \mathrm{~N}}$ right after the step is given by

$$
\mathrm{P}_{\gamma}\left(r^{3 \mathrm{~N}}\right)=\frac{\exp \left\{-\beta \Phi\left(\vec{r}_{1}-\gamma y_{1} \widehat{e}_{z}, \ldots, \vec{r}_{\mathrm{N}}-\gamma y_{\mathrm{N}} \widehat{e}_{z}\right)\right\}}{Z_{\gamma}}
$$

where $Z_{\gamma}$ is the normalizing constant or partition function and $\beta=\left(k_{\mathrm{B}} \mathrm{T}\right)^{-1}$. Expanding $\Phi$ to first order in $\gamma$ and next the exponential, we obtain

$$
\mathrm{P}_{\gamma}\left(r^{3 \mathrm{~N}}\right)=\frac{\exp \left\{-\beta \Phi\left(r^{3 \mathrm{~N}}\right)\right\}}{\mathrm{Z}_{\gamma}}\left(1+\beta \gamma \mathrm{V} \sigma_{y z}\left(r^{3 \mathrm{~N}}\right)\right) .
$$

Next we calculate the partition function

$$
\begin{aligned}
Z_{\gamma} & =\int d^{3 \mathrm{~N}} \exp \left\{-\beta \Phi\left(r^{3 \mathrm{~N}}\right)\right\}\left(1+\beta \gamma \mathrm{V} \sigma_{y z}\left(r^{3 \mathrm{~N}}\right)\right) \\
& =\mathrm{Z}\left(1+\beta \gamma \mathrm{V}\left\langle\sigma_{y z}\right\rangle\right),
\end{aligned}
$$

where the angular brackets indicate an equilibrium average. Using that the average stress is zero at equilibrium, we find

$$
\mathrm{P}_{\gamma}\left(r^{3 \mathrm{~N}}\right)=\mathrm{P}_{e q}\left(r^{3 \mathrm{~N}}\right)\left(1+\beta \gamma \mathrm{V} \sigma_{y z}\left(r^{3 \mathrm{~N}}\right)\right),
$$

where $\mathrm{P}_{e q}\left(r^{3 \mathrm{~N}}\right)=\exp \left\{-\beta \Phi\left(r^{3 \mathrm{~N}}\right)\right\} / \mathrm{Z}$. Denoting the stress at time $t$ of a system that at time zero had the configuration $r^{3 \mathrm{~N}}$ by $\sigma_{y z}\left(t ; r^{3 N}\right)$, we obtain for the modulus

$$
\begin{aligned}
G^{\mathrm{S}}(\mathrm{t}) & =\frac{1}{\gamma} \int d^{3 \mathrm{~N}} r \sigma_{y z}\left(t ; r^{3 \mathrm{~N}}\right) \mathrm{P}_{\gamma}\left(r^{3 \mathrm{~N}}\right) \\
& =\beta \mathrm{V} \int d^{3 \mathrm{~N}} r \mathrm{P}_{e q}\left(r^{3 \mathrm{~N}}\right) \sigma_{y z}\left(t ; r^{3 \mathrm{~N}}\right) \sigma_{y z}\left(r^{3 \mathrm{~N}}\right),
\end{aligned}
$$

where we have used that $\left\langle\sigma_{y z}(t)\right\rangle=0$. This result may be written in its usual notation as

$$
G^{s}(t)=\frac{V}{k_{B} T}\left\langle\sigma_{y z}(t) \sigma_{y z}(0)\right\rangle \text {. }
$$

In equilibrium, when the modulus after a strain step is the same as the one from oscillatory experiments, we may remove the superscript "s."

\section{REFERENCES}

${ }^{1}$ H. Booij, Rheol. Acta 5, 215 (1966).

${ }^{2} \mathrm{~J}$. Vermant, L. Walker, P. Moldenaers, and J. Mewis, J. Non-Newtonian Fluid Mech. 79, 173 (1998).

${ }^{3}$ J. K. Dhont and N. J. Wagner, Phys. Rev. E 63, 021406 (2001).

${ }^{4}$ R. Tanner and J. Simmons, Chem. Eng. Sci. 22, 1803 (1967).

${ }^{5}$ A. Leonov, E. Lipkina, E. Paskhin, and A. Prokunin, Rheol. Acta 15, 411 (1976).

${ }^{6}$ R. Tanner and G. Williams, Trans. Soc. Rheol. 14, 19 (1970).

${ }^{7}$ R. Tanner and G. Williams, Rheol. Acta 10, 528 (1971).

${ }^{8}$ M. Yamamoto, Trans. Soc. Rheol. 15, 331 (1971).

${ }^{9} \mathrm{G}$. De Cleyn and J. Mewis, J. Non-Newtonian Fluid Mech. 9, 91 (1981).

10J. Simmons, Rheol. Acta 7, 184 (1968).

${ }^{11}$ K. Osaki, M. Tamura, M. Kurata, and T. Kotaka, J. Phys. Chem. 69, 4183 (1965).

${ }^{12}$ T. Kitano, S. Hashmi, and N. Chand, Bull. Mater. Sci. 27, 409 (2004).

${ }^{13}$ G. Vlastos, D. Lerche, B. Koch, O. Samba, and M. Pohl, Rheol. Acta 36, 160 (1997).

${ }^{14}$ B. d. L. Costello, J. Non-Newtonian Fluid Mech. 68, 303 (1997).

${ }^{15}$ E. Somma, O. Valentino, G. Titomanlio, and G. Ianniruberto, J. Rheol. 51, 987 (2007).

${ }^{16}$ A. Isayev and C. Wong, J. Polym. Sci., Part B: Polym. Phys. 26, 2303 (1988).

${ }^{17}$ S. Khandavalli, J. Hendricks, C. Clasen, and J. P. Rothstein, J. Rheol. 60, 1331 (2016).

${ }^{18}$ A. R. Jacob, A. S. Poulos, S. Kim, J. Vermant, and G. Petekidis, Phys. Rev. Lett. 115, 218301 (2015).

${ }^{19}$ A. Van Den Noort and W. J. Briels, Macromol. Theory Simul. 16, 742 (2007).

${ }^{20} \mathrm{Z}$. Laufer, H. Jalink, and A. Staverman, Rheol. Acta 14, 641 (1975).

${ }^{21}$ Z. Laufer, H. L. Jalink, and A. J. Staverman, Rheol. Acta 14, 650 (1975).

${ }^{22}$ D. Curtis, N. Badiei, A. Holder, J. Claypole, D. Deganello, M. Brown, M. Lawrence, P. Evans, P. Williams, and K. Hawkins, J. Non-Newtonian Fluid Mech. 222, 227 (2015).

${ }^{23}$ P. Ballesta, M. P. Lettinga, and S. Manneville, J. Rheol. 51, 1047 (2007).

24J. Simmons, J. Sci. Instrum. 43, 887 (1966).

${ }^{25}$ T. Jones and K. Walters, J. Phys. A: Gen. Phys. 4, 85 (1971).

${ }^{26}$ B. Bernstein, Rheol. Acta 11, 210 (1972).

${ }^{27}$ L. M. Walker, J. Vermant, P. Moldenaers, and J. Mewis, Rheol. Acta 39, 26 (2000).

${ }^{28} \mathrm{~J}$. Zeegers, D. van den Ende, C. Blom, E. G. Altena, G. J. Beukema, and J. Mellema, Rheol. Acta 34, 606 (1995).

${ }^{29} \mathrm{~J}$. Vermant, P. Moldenaers, J. Mewis, M. Ellis, and R. Garritano, Rev. Sci. Instrum. 68, 4090 (1997).

${ }^{30} \mathrm{~J}$. Mewis, B. Kaffashi, J. Vermant, and R. Butera, Macromolecules 34, 1376 (2001).

${ }^{31}$ G. Colombo, S. Kim, T. Schweizer, B. Schroyen, C. Clasen, J. Mewis, and J. Vermant, J. Rheol. 61, 1035 (2017).

${ }^{32}$ C. Gracia-Fernández, S. Gómez-Barreiro, A. Elmoumni, A. Álvarez, J. López-Beceiro, and R. Artiaga, J. Rheol. 60, 121 (2016).

${ }^{33}$ N. G. van Kampen, J. Phys. Radium 22, 179 (1961).

${ }^{34}$ T. F. Farage and J. M. Brader, J. Rheol, 56, 259 (2012).

${ }^{35}$ M. Fuchs and M. E. Cates, Phys. Rev. Lett. 89, 248304 (2002).

${ }^{36}$ M. Fuchs and M. E. Cates, J. Rheol. 53, 957 (2009).

${ }^{37}$ D. Vlassopoulos, G. Fytas, T. Pakula, and J. Roovers, J. Phys.: Condens. Matter 13, R855 (2001). 
${ }^{38}$ C. Likos, H. Löwen, M. Watzlawek, B. Abbas, O. Jucknischke, J. Allgaier, and D. Richter, Phys. Rev. Lett. 80, 4450 (1998).

${ }^{39}$ T. G. Mason and D. Weitz, Phys. Rev. Lett. 74, 1250 (1995).

${ }^{40}$ R. M. Cristensen, Theory of Viscoelasticity (Dover Publications, 2003).

${ }^{41}$ W. Briels, Han-sur-Lesse Winterschool Notes (2009).

${ }^{42}$ D. Forster, Hydrodynamic Fluctuations, Broken Symmetry, and Correlation Functions (W.A. Benjamin, 1975).

${ }^{43}$ V. Metri, A. Louhichi, J. Yan, G. P. Baeza, K. Matyjaszewski, D. Vlassopoulos, and W. J. Briels, Macromolecules 51, 2872 (2018).

${ }^{44} \mathrm{~F}$. Schwarzl, Pure Appl. Chem. 23, 219 (1970).

${ }^{45} \mathrm{H}$. Jin, K. Kang, and J. K. Dhont, Soft Matter 10, 9470 (2014).
${ }^{46}$ S. Kim, J. Mewis, C. Clasen, and J. Vermant, Rheol. Acta 52, 727 (2013).

${ }^{47} \mathrm{~V}$. Breedveld, D. van den Ende, R. Jongschaap, and J. Mellema, J. Chem. Phys. 114, 5923 (2001).

${ }^{48} \mathrm{H}$. Markovitz, Journal of Polymer Science: Polymer Symposia (Wiley Online Library, 1975), Vol. 50, pp. 431-456.

${ }^{49} \mathrm{~S}$. Milner and T. McLeish, Macromolecules 30, 2159 (1997).

${ }^{50}$ S. Milner and T. McLeish, Macromolecules 31, 7479 (1998).

${ }^{51}$ P. Kindt and W. J. Briels, J. Chem. Phys. 127, 134901 (2007).

${ }^{52}$ L. Liu, W. K. den Otter, and W. J. Briels, Soft Matter 10, 7874 (2014).

${ }^{53}$ G. Ianniruberto, A. Brasiello, and G. Marrucci, Macromolecules 45, 8058 (2012). 